\title{
Determinación del mercado objetivo y la demanda insatisfecha, cuando no se dispone de estadísticas
}

Carlos Izquierdo Maldonado*

\begin{abstract}
Resumen
El plan de negocios y/o proyecto de factibilidad representa la mejor alternativa de inversión con un nivel de rentabilidad mayor al costo de oportunidad del capital. Para que el retorno del proyecto sea mayor al costo de oportunidad, esto significa sustentar los flujos de beneficios que ofrece el proyecto, con la determinación de la demanda insatisfecha y un desempeño eficiente del negocio; consecuentemente, determinar la demanda insatisfecha es un elemento clave para demostrar la factibilidad del proyecto. No siempre se dispone de estadísticas para el estudio de mercado de nuevas oportunidades de negocios; en tal sentido, se ha preparado esta metodología, con base en un estudio de caso para la determinación del mercado objetivo y demanda insatisfecha cuando no se dispone de estadísticas.
\end{abstract}

\section{Palabras clave}

Proyecto de factibilidad, plan de negocios, mercado objetivo, demanda insatisfecha sin estadísticas

\begin{abstract}
The business plan and / or feasibility study represents the best investment alternative, with a level of return greater than the opportunity cost of capital. When the return of the project is greater than the opportunity cost, means sustaining the flow of benefits of the project, with the determination of unmet demand and an efficient performance of the project, and consequently, determine the unmet demand is a key element to show the feasibility of the project. There is not always available statistics for the market research of new business opportunities, in this sense, we have developed this methodology, based on a case study for the determination of the target market and unmet when there are no statistics.
\end{abstract}

\section{Key words}

Project feasibility, business plan, target market, no statistical unmet demand

Forma sugerida de citar: Izquierdo, Carlos. 2011. Determinación del mercado objetivo y la demanda insatisfecha, cuando no se dispone de estadísticas. Retos 1. Enero/Junio. Pp. 41-52.

\footnotetext{
* Docente de la Universidad Politécnica Salesiana. Carrera de Administración de Empresas-Sede Quito. cizquierdo@ups.edu.ec.
}

Retos I(I): 20II.

(c) 2011, Universidad Politécnica Salesiana del Ecuador 


\section{¿Qué es un proyecto de factibilidad?}

Es una alternativa de inversión determinada por el retorno de lo invertido, que cuenta con los medios necesarios para asegurar los resultados esperados, y tiene como base el método científico para sustentar y demostrar la viabilidad del proyecto, con sus componentes: mercado, estudio técnico, financiero, gestión y medioambiente. Equivale a planificar la viabilidad de un negocio o empresa.

\section{Finalidad del estudio de factibilidad}

El proyecto es un instrumento financiero que sirve para tomar la decisión de invertir en determinada actividad, en función del rendimiento y nivel de riesgo. Consecuentemente, el estudio de factibilidad constituye el principal requisito para aprobar su financiamiento. Por otra parte, el proyecto de factibilidad es una de las modalidades de tesis o trabajo de grado para optar por el título de Ingeniero Comercial en la UPS.

\section{¿Cómo sustentar y demostrar la viabilidad de un negocio, sin contar con estadísticas?}

Las instituciones públicas y privadas encargadas de la generación, procesamiento y publicación de datos estadísticos, por lo general centran su atención en las actividades de mayor dinamismo de la economía. Esta situación dificulta poder realizar estudios de factibilidad de bienes y servicios, porque estos no cuentan con datos estadísticos.

Al no contar con estos, necesariamente se tiene que generar o levantar los datos de fuentes primarias, en particular datos e informaciones que se requieren en el estudio de mercado: número de compradores, cantidades consumidas, precios, competidores, etcétera.

Se ha desarrollado una serie de pasos, de orden metodológico, para determinar el mercado objetivo y la demanda insatisfecha de un producto - en este caso: el amaranto reventado, que no cuenta con estadísticas y publicaciones.

Con esta metodología se espera dotar a los estudiantes de una herramienta para la planificación y desarrollo de sus trabajos de grado.

\section{AMARANTO REVENTADO}

(Tipo flakes o granola)

\section{Definición del producto y ventaja competitiva}

Es un cereal reventado, tipo flakes o granola, que viene en fundas de polietileno y empacado en cajas de cartón de 500 gramos, mezclado con hojuelas de avena, nueces y miel de panela y listo para su consumo. Es un producto sano, limpio y de sabor agradable, con un alto contenido de lisina del 16,6\%, aminoácido esencial que se encuentra en la leche en una proporción del 16,5\%, ideal para anemias, desnutrición y osteoporosis; de consumo directo al agregarlo a jugos y postres; dirigido a la población 
de ingresos medios-altos, por sus hábitos de consumo, calidad y precio.

\section{Datos básicos}

Se deben recoger datos básicos de las características físicas, químicas y beneficios del producto; precios, estándares de calidad, tecnología de producción y distribución; productos sustitutos, complementarios e insumos, en el país y otros mercados internacionales.

Para la segmentación de mercados es necesario recopilar datos demográficos del INEC; en este caso, de la pobla- ción urbana de la ciudad de Quito, por grupos de edad y niveles de ingresos.

\section{Muestra piloto}

Para determinar el número de compradores potenciales, es necesario estructurar y aplicar una muestra piloto mayor a 50 datos, a través de diferentes medios: entrevistas telefónicas o encuestas dirigidas al segmento de mercado, a domicilio o en lugares de gran afluencia del público.

Es relevante recoger datos básicos como los siguientes:

\begin{tabular}{|c|c|c|}
\hline ¿Consume el producto? & sí & NO (razones) \\
\hline Si consume el producto está & $\begin{array}{l}\text { SATISFECHO } \\
\text { (razones) }\end{array}$ & $\begin{array}{l}\text { NO SATISFECHO } \\
\text { (razones) }\end{array}$ \\
\hline $\begin{array}{l}\text { Si está satisfecho, ¿se cambiaría por un mejor produc- } \\
\text { to? }\end{array}$ & sí & NO \\
\hline $\begin{array}{l}\text { Si no está satisfecho, ¿se cambiaría por un mejor } \\
\text { producto? }\end{array}$ & sí & NO \\
\hline $\begin{array}{l}\text { Si no consume, ¿adquiriría un producto de calidad } \\
\text { que satisfaga sus necesidades? }\end{array}$ & Sí & NO \\
\hline
\end{tabular}

\section{Mercado objetivo}

Es un conjunto de clientes bien definido, cuyas necesidades planea satisfacer la empresa.

Para nuestro caso, el mercado objetivo lo constituyen los compradores atendidos por la competencia (satisfechos e insatisfechos), que estarían dispuestos a cambiarse por un producto y beneficios superiores. Asimismo, el mercado objetivo también lo constituyen aquellas personas y agentes que no lo están comprando actualmente y que, al conocer las bondades y beneficios del nuevo producto, tienen interés por adquirirlo.

Retos I(I): 20I I.

(C) 201 I, Universidad Politécnica Salesiana del Ecuador
Con base en los datos recogidos de las estadísticas demográficas y de la muestra piloto, se procede a definir el mercado objetivo, para determinar el número de compradores potenciales insatisfechos.

El mercado objetivo sirve, además, para determinar el tamaño de la muestra, el diseño del formato de encuesta y su aplicación.

Una vez tabulados los datos de la muestra, de existir divergencias significativas entre los resultados de la muestra y el mercado objetivo, se procederá a actualizar las variables y magnitudes del mercado objetivo. 
Determinación del mercado objetivo y la demanda insatisfecha, cuando no se dispone de estadísticas

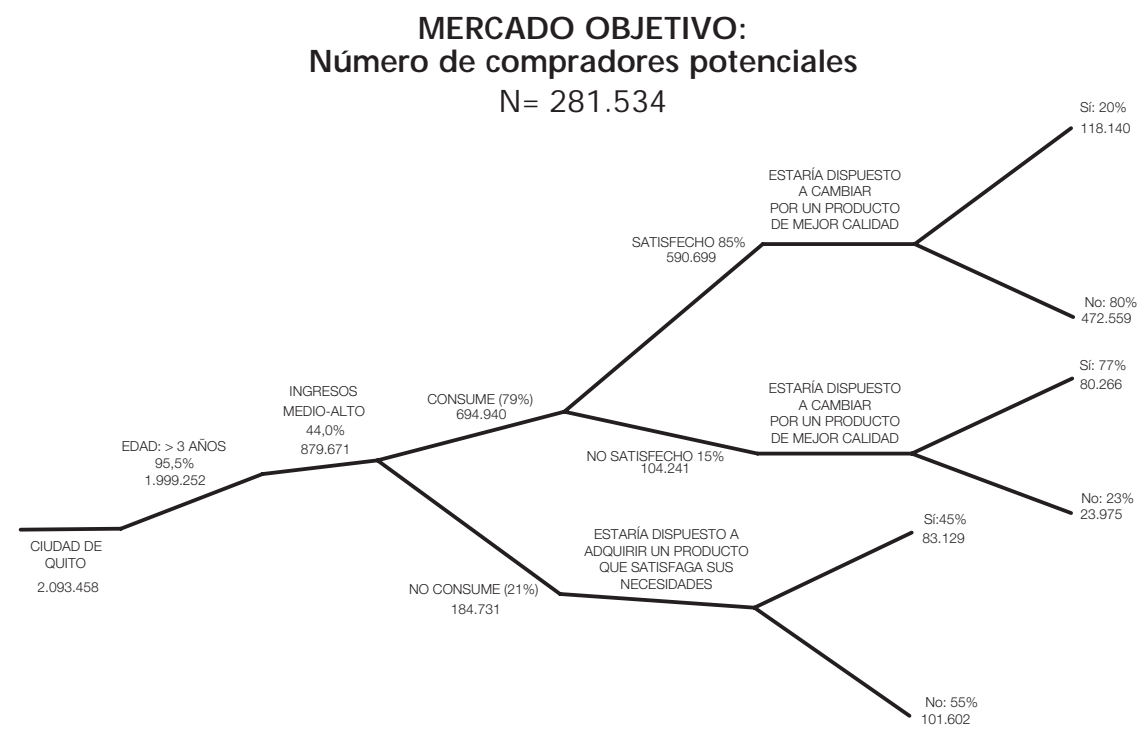

MERCADO OBJETIVO

\begin{tabular}{|c|c|c|}
\hline COMPRADORES POTENCIALES ACTUALES & & 778.069 \\
\hline ATENDIDOS SATISFECHOS & 590.699 & \\
\hline NO ATENDIDOS INSATISFECHOS & 104.241 & \\
\hline ATENDIDOS NO SATISFECHOS & 83.129 & \\
\hline COMPRADORES ATENDIDOS (OFERTA) & & 496.535 \\
\hline ATENDIDOS SATISFECHOS NO DESEAN CAMBIARSE & 472.559 & \\
\hline ATENDIDOS NO SATISFECHOS NO DESEAN CAMBIARSE & 23.975 & \\
\hline MERCADO OBJETIVO INSATISFECHO (N) & & 281.534 \\
\hline ATENDIDOS SATISFECHOS POR CAMBIARSE & 118.140 & \\
\hline ATENDIDOS NO SATISFECHOS POR CAMBIARSE & 80.266 & \\
\hline NO ATENDIDA INSATISFECHA & 83.129 & \\
\hline
\end{tabular}

Fuente: Actualización de datos del "Estudio de factibilidad para la creación de una empresa dedicada a la producción y comercialización de amaranto reventado en la ciudad de Quito", Tesis de Grado Ing. Comercial UPSQ, 2010.

Así determinado, el mercado objetivo es de $\mathbf{2 8 1 . 5 3 4}$ compradores potenciales.

\section{Tamaño de la muestra $(\mathrm{N})$}

Existen diferentes métodos para determinar el tamaño de la muestra, dependiendo principalmente de la disponibilidad de información.

Retos I(I): 201I.

C) 20II, Universidad Politécnica Salesiana del Ecuador
Al tener una muestra piloto y conocer la media y la desviación estándar, es recomendable aplicar el método de la media, que determina un tamaño representativo de la muestra.

Al no conocer la media y la desviación estándar de la muestra piloto, se puede aplicar el método de las proporciones, por su facilidad de cálculo, 
pero determina un tamaño de muestra mayor.

Tamaño de Muestra (n)

$$
n=\frac{N^{\star} Z^{\wedge} " 2 P m^{*} Q m}{Z^{\wedge} 2^{*} P m^{*} Q m+(N-1)(E p)^{\wedge} 2}
$$

Nivel de confianza: 95\% $=>\begin{aligned} & Z \pm 1,96 \\ & E=0,05\end{aligned}$

$$
\mathrm{n}=\frac{879.671^{\star}(1,96)^{\wedge} 2^{\star}(0,5)(0,5)}{(1,96)^{\wedge} 2^{\star}(0,5)(0,5)+(\mathrm{N}-1)(0,05)^{\wedge} 2}=384
$$

\section{Diseño del formato de encuesta}

Una de las etapas más difíciles de la investigación de mercado es la del diseño del formato de encuesta, pues no está muy claro qué datos cualitativos y cuantitativos necesitamos recoger.

Criterios generales para diseñar el formato de encuesta:

\section{Objetivos e hipótesis del estudio de mercado}

Son los que orientan y delimitan el tipo de datos que necesitamos recoger.
Si los objetivos de mercado se orientan a determinar la cantidad de la demanda insatisfecha y a definir las estrategias de mercadeo para penetrar en él, en consideración a las fuerzas de la competencia y la capacidad de la empresa, entonces, las preguntas de la encuesta deben estructurarse en función de tales objetivos. Es decir, de los objetivos de mercado se deriva el número de compradores potenciales, el consumo per-cápita y la demanda insatisfecha $(\mathrm{N} x \mathrm{Cpc})$.

\section{Frecuencia de consumo}

Para determinar el consumo anual total y per-cápita, se registran las cantidades demandadas basados en su frecuencia, que puede ser: diaria, semanal, quincenal, mensual y ocasional, y se actualizan en la tabulación.

\section{Prueba de la encuesta}

Una vez estructurado el cuestionario de encuesta es necesario realizar algunas pruebas y ajustes al formato, para obtener una mayor claridad de las preguntas, la debida secuenciación y una comprensión uniforme por parte de los encuestados. 
Determinación del mercado objetivo y la demanda insatisfecha, cuando no se dispone de estadísticas

\section{Mercado objetivo o compradores insatisfechos de la muestra}

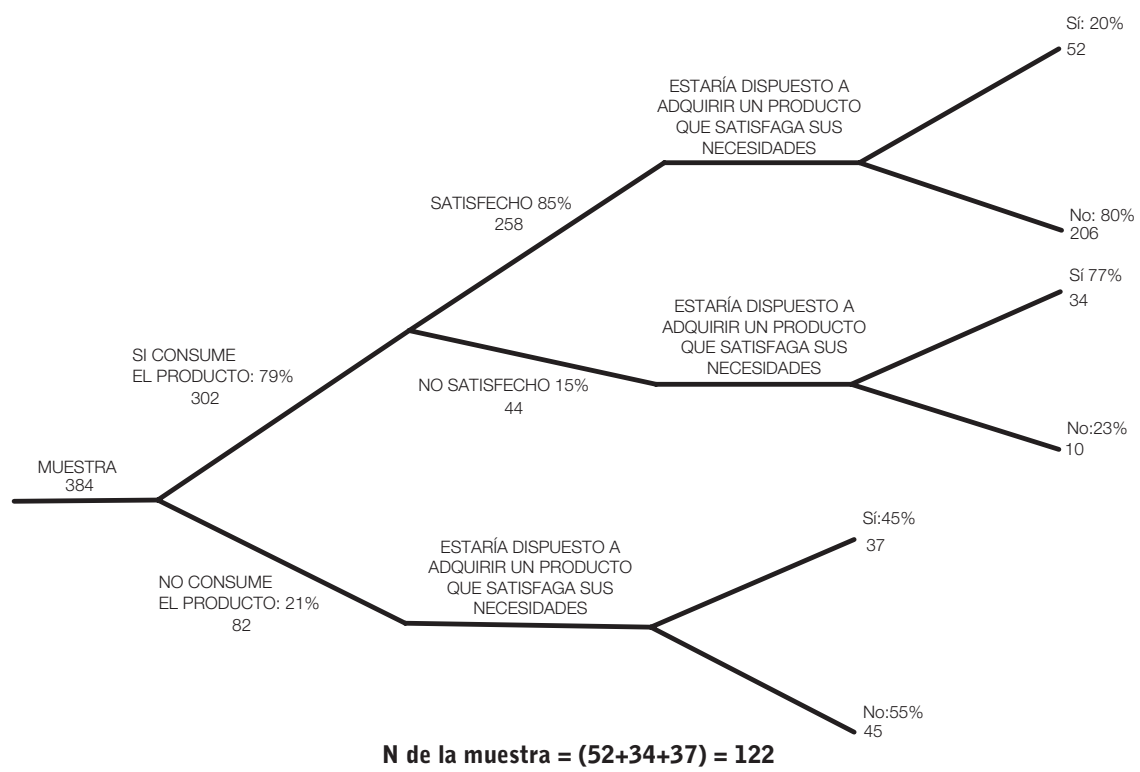

MERCADO OBJETIVO INSATISFECHO DE LA MUESTRA

COMPRADORES POTENCIALES ACTUALES

ATENDIDOS SATISFECHOS 258

NO ATENDIDOS INSATISFECHOS

44

ATENDIDOS NO SATISFECHOS

37

COMPRADORES ATENDIDOS (OFERTA)

ATENDIDOS SATISFECHOS NO DESEAN CAMBIARSE

206

ATENDIDOS NO SATISFECHOS NO DESEAN CAMBIARSE

10

MERCADO OBJETIVO INSATISFECHO (N)

ATENDIDOS SATISFECHOS POR CAMBIARSE

ATENDIDOS NO SATISFECHOS POR CAMBIARSE

NO ATENDIDA INSATISFECHA

Fuente: Ibídem 


\section{Consumo per-cápita}

La tabulación del consumo total y per-cápita de las 302 encuestas de la muestra, que contestaron que sí consumían amaranto reventado tipo granola, se presenta en el cuadro siguiente.

CONSUMO PER-CÁPITA (Cpc)

\begin{tabular}{|c|c|c|c|c|}
\hline FRECUENCIA & g & Días/año & Frecuencia & g/año \\
\hline 1 vez por semana & 90 & 52 & 20 & 93.600 \\
\hline 1 vez por semana & 120 & 52 & 45 & 280.800 \\
\hline 1 vez por semana & 150 & 52 & 30 & 234.000 \\
\hline 2 veces por semana & 125 & 52 & 10 & 65.000 \\
\hline 2 veces por semana & 185 & 52 & 22 & 211.640 \\
\hline 2 veces por semana & 225 & 52 & 18 & 210.600 \\
\hline 1 cada 15 días & 155 & 26 & 18 & 72.540 \\
\hline 1 cada 15 días & 175 & 26 & 34 & 154.700 \\
\hline 1 cada 15 días & 200 & 26 & 30 & 156.000 \\
\hline $1 \mathrm{vez}$ al mes & 137 & 12 & 35 & 57.540 \\
\hline 1 vez al mes & 164 & 12 & 25 & 49.200 \\
\hline 1 vez al mes & 205 & 12 & 15 & 36.900 \\
\hline \multicolumn{3}{|c|}{ TOTAL MUESTRA } & 302 & 1.622 .520 \\
\hline \multicolumn{4}{|c|}{ Cpc/g/año } & 5.373 \\
\hline \multicolumn{4}{|c|}{ Cpc/kg/año 2010} & 5,4 \\
\hline \multicolumn{4}{|c|}{ Cpc/kg/año 2009} & 5,3 \\
\hline
\end{tabular}

Fuente: Ibídem

El Cpc de amaranto reventado en el año 2010, es de 5,4 kg.

\section{Demanda actual insatisfecha (DI)}

La DI, producto de multiplicar el número de compradores potenciales por el consumo per-cápita $(\mathrm{N}$ x $\mathrm{Cpc})$, es de $1.520 .284 \mathrm{~kg} / \mathrm{año}$, correspondiente al año de investigación (2010).

\section{DEMANDA ACTUAL} INSATISFECHA (DI)

$$
\begin{aligned}
& \mathrm{DI}=\mathrm{N} \times \mathrm{Cpc} \\
& \mathrm{DI}=281.534 \times 5,4 \\
& \mathrm{DI}=\mathbf{1 . 5 2 0 . 2 8 4} \mathrm{kg} / \mathrm{año}
\end{aligned}
$$

Retos I(I): 20II

(C) 201 I, Universidad Politécnica Salesiana del Ecuador

\section{Demanda histórica insatisfecha}

El no contar con datos históricos del consumo dificulta elaborar la serie histórica del consumo de amaranto reventado, en un período de al menos cinco años.

En este caso concreto se planteó dos preguntas: ¿cuál fue la frecuencia de consumo en gramos el año actual? y ¿cuál el año pasado?

Como la demanda resulta de multiplicar N x Cpc, para los años 20082007-2006, se tomó el Cpc del año 
Determinación del mercado objetivo y la demanda insatisfecha, cuando no se dispone de estadísticas

2009 de 5,3 kg/año. La N de la población o compradores potenciales insatisfechos se estimó con base en la tasa de crecimiento vegetativo de la población de 2,3\% anual.

\section{DEMANDA HISTÓRICA}

\begin{tabular}{cc|}
\hline AÑOS & $\begin{array}{c}\text { DEMANDA INSATIFECHA } \\
(\mathbf{k g} / \mathrm{año})\end{array}$ \\
\hline \multirow{2}{*}{2006} & 1.344 .214 \\
& $\left(262.552^{*} 0,977\right)^{\star} 5,3$ \\
2007 & 1.391 .521 \\
& $\left(268.732^{*} 0,977\right)^{\star} 5,3$ \\
2008 & 1.424 .283 \\
& $\left(275059^{\star} 0,977\right)^{\star} 5,3$ \\
2009 & 1.457 .811 \\
& $\left(281.534^{*} 0,977\right)^{\star} 5,3$ \\
2010 & 1.520 .284 \\
& $281.534^{*} 5,4$
\end{tabular}

\section{Función de proyección}

Para determinar el tipo de función de proyección o de ajuste, se tiene que analizar el comportamiento de los datos históricos que grafica la variable dependiente de consumo (Y), determinada por la variación de la variable independiente del tiempo (X).

En el presente caso, para la proyección de la demanda insatisfecha se aplican dos funciones matemáticas: la función lineal y la función exponencial.

Se selecciona la función de proyección exponencial:

$$
y=(1.306 .465,9)(1,0297)^{\wedge} x
$$

Debido a que el coeficiente de correlación de 0,99 se acerca a 1, frente el coeficiente de correlación de 0,90 de la función lineal.

\section{Función de proyección lineal}

\section{Función Lineal}

$$
\begin{aligned}
& y=f(x) \\
& y=\text { consumo de amaranto } \\
& X=\text { años } \\
& Y=a+b X
\end{aligned}
$$

La función de proyección se obtiene determinando los parámetros a y b a partir de los datos históricos de la variable dependiente del consumo, en función de las variaciones de la variable independiente $\mathrm{X}$.

\section{Método: Mínimos Cuadrados}

$$
\begin{aligned}
& y=a+b x \\
& \square y=n a+b \square x \\
& \square x y=a \square x+b \square x^{\wedge} 2(2): \quad a(1)^{\star} x
\end{aligned}
$$

\begin{tabular}{|c|c|c|c|c|}
\hline AÑOS & AÑOS & DEMANDA & & \\
\hline & $X$ & $Y$ & $X Y$ & $X^{\wedge} 2$ \\
\hline 2006 & 1 & 1.344 .214 & 1.344 .214 & 1 \\
\hline 2007 & 2 & 1.391 .521 & 2.783 .042 & 4 \\
\hline 2008 & 3 & 1.424 .283 & 4.272 .849 & 9 \\
2009 & 4 & 1.457 .811 & 5.831 .245 & 16 \\
\hline 2010 & 5 & 1.520 .284 & 7.601 .418 & 25 \\
\hline$\square$ & 15 & 7.138 .113 & 21.832 .768 & 55 \\
\hline
\end{tabular}

Retos I(I): $201 \mathrm{I}$.

(C) 2011, Universidad Politécnica Salesiana del Ecuador 


$$
\begin{aligned}
& \mathrm{n}=5 \\
& 7.138 .113=5 a+15 b \\
& \text { (1) } \quad(1)^{\star}-3 \\
& 21.832 .768=15 \mathrm{a}+55 \mathrm{~b} \\
& \text { (2) } \\
& -21.414 .338=-15 a-45 b \\
& 21.414 .338=15 a+55 b \\
& 418.430=10 \mathrm{~b} \\
& \mathrm{~b}=\frac{418.430}{10} \\
& \text { b }=41.842,99
\end{aligned}
$$

\begin{tabular}{|c|c|c|c|c|c|c|}
\hline$x$ & Y & $(Y-\tilde{y})$ & $(Y-\tilde{y})^{\wedge} 2$ & $Y^{\prime}$ & $\left(Y^{\prime}-\tilde{y}\right)$ & $\left(Y^{\prime}-\tilde{y}\right)^{\wedge} 2$ \\
\hline 1 & 1.344 .214 & -83.409 & 6.957 .030 .056 & 1.384 .387 & -43.236 & 1.869 .308 .670 \\
\hline 2 & 1.391 .521 & -36.101 & 1.303 .309 .148 & 1.418 .164 & -9.459 & 89.473 .484 \\
\hline 3 & 1.424 .283 & -3.340 & 11.152 .491 & 1.451 .940 & 24.317 & 591.336 .798 \\
\hline 4 & 1.457 .811 & 30.189 & 911.355 .373 & 1.485 .716 & 58.094 & 3.374.898.612 \\
\hline 5 & 1.520 .284 & 92.661 & 8.586 .071 .592 & 1.519 .493 & 91.870 & 8.440 .158 .927 \\
\hline 15 & 7.138 .113 & 0 & 17.768 .918 .660 & & & 14.365 .176 .490 \\
\hline
\end{tabular}

En (1) reemplazamos $\mathbf{b}$

$$
\begin{array}{rcccc}
7.138 .113 & = & 5 a & + & 15(41.842,99) \\
7.138 .113 & = & 5 a & & 627.644,80 \\
a & = & \frac{6.510 .467 .92}{5} & & \\
a & = & 1.302 .093,58 & &
\end{array}
$$

\section{FUNCIÓN DE PROYECCIÓN O AJUSTE}

$$
Y=1.302 .093,58+41.842,99 X
$$

\section{COEFICIENTE DE DETERMINACIÓN R^2}

$\mathrm{R}^{\wedge} 2=$ VARIACIÓN EXPLICADA POR LA FUNCIÓN DE AJUSTE VARIACIÓN TOTAL

$R \wedge 2=\frac{\sum\left(Y^{\prime}-\tilde{Y}\right) \wedge 2}{\sum(Y-\tilde{Y}) \wedge 2}$

\section{AÑOS DEMANDA}


Determinación del mercado objetivo y la demanda insatisfecha, cuando no se dispone de estadísticas

\begin{tabular}{|c|c|c|c|c|c|}
\hline \multirow[b]{2}{*}{$\frac{\sum\left(Y^{\prime}-\tilde{y}\right) \wedge 2}{\sum(Y-\tilde{y}) \wedge 2}$} & $=$ & $\frac{\Sigma Y}{n}$ & $=$ & $\frac{7.138 .113}{5}$ & \multirow[t]{4}{*}{$=1.427 .623$} \\
\hline & $=$ & $\frac{14.365 .176 .490}{17.768 .918 .660}$ & $=$ & 0,8084 & \\
\hline$r$ & $=$ & $\sqrt{R \wedge} 2$ & & & \\
\hline$r$ & $=$ & 0,90 & & & \\
\hline
\end{tabular}

\section{PROYECCIÓN DE LA DEMANDA INSATISFECHA}

\begin{tabular}{|ccc|}
\hline AÑOS & AÑOS X & DEMANDA PROYECTADA Y' \\
\hline 2011 & 6 & 1.553 .152 \\
2012 & 7 & 1.594 .995 \\
2013 & 8 & 1.636 .838 \\
2014 & 9 & 1.678 .680 \\
2015 & 10 & 1.720 .523 \\
\hline
\end{tabular}

\section{Función exponencial}

\begin{tabular}{c|cccc}
\hline AÑOS & DEMANDA & & & \\
\hline$X$ & $Y$ & $\ln Y$ & $X \ln Y$ & $X^{\wedge} 2$ \\
\hline 1 & 1.344 .214 & 14,11131981 & 14,11131981 & 1 \\
\hline 2 & 1.391 .521 & 14,14590807 & 28,29181615 & 4 \\
\hline 3 & 1.424 .283 & 14,16917909 & 42,50753728 & 9 \\
\hline 4 & 1.457 .811 & 14,19244669 & 56,76978678 & 16 \\
\hline 5 & 1.520 .284 & 14,23440745 & 71,17203727 & 25 \\
\hline 15 & 7.138 .113 & 70,85326113 & 212,8524973 & 55 \\
\hline
\end{tabular}

$$
\begin{aligned}
& y=f(x) \\
& y=\text { consumo de amaranto } \\
& \mathrm{x}=\text { años } \\
& \ln Y=\ln a+X \ln b \\
& \Sigma \operatorname{lny}=n \operatorname{lna}+\Sigma X \mid n b \\
& \Sigma \ln Y=\Sigma X \mid n a+\Sigma x^{\wedge} 2 \ln b \\
& \text { (2):(1)*x } \\
& \text { Media }=\frac{7.138 .113}{5}=1.427 .623
\end{aligned}
$$

\section{Ecuaciones}




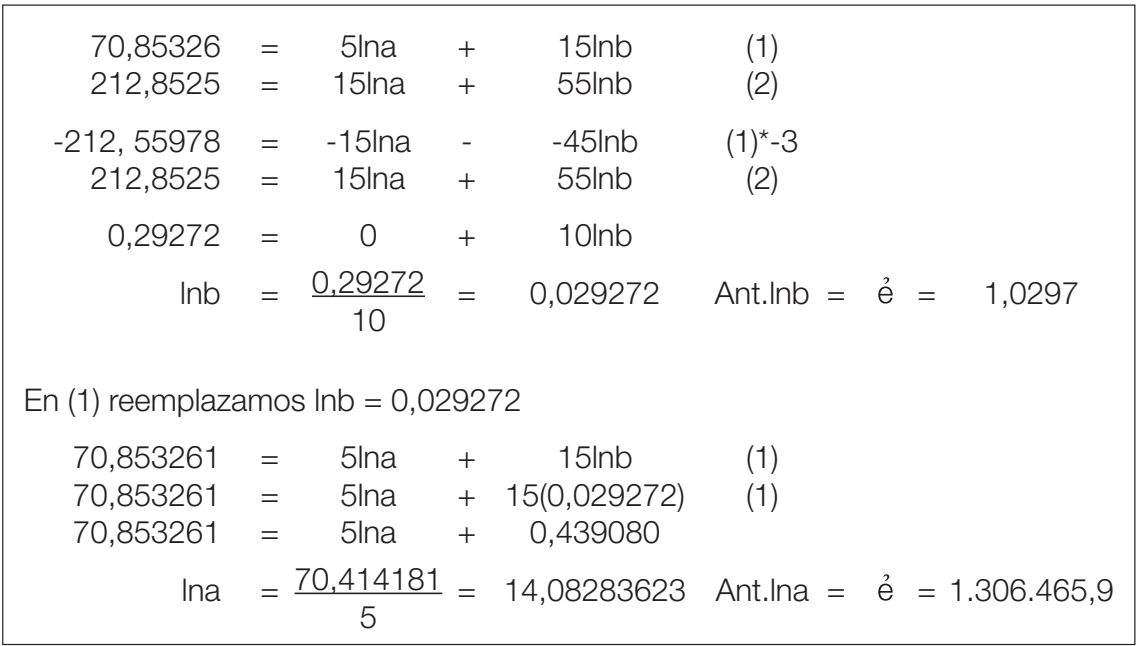

\section{FUNCIÓN DE PROYECCIÓN \\ $Y=(1.306 .465,9)(1,0297)^{\wedge} x$}

\section{COEFICIENTE DE DETERMINACIÓN R^2}

R^2 = VARIACIÓN EXPLICADA POR LA FUNCIÓN DE AJUSTE VARIACIÓN TOTAL

$$
R^{\wedge} 2=\frac{\sum\left(Y^{\prime}-\tilde{y}\right) \wedge 2}{\sum(Y-\tilde{y}) \wedge 2}
$$

\section{AÑOS DEMANDA}

\begin{tabular}{|c|c|c|c|c|c|c|}
\hline$x$ & Y & $(Y-\tilde{y})$ & $(Y-\bar{y})^{\wedge} 2$ & $Y^{\prime}$ & $\left(Y^{\prime}-\tilde{y}\right)$ & $\left(Y^{\prime}-\tilde{y}\right)^{\wedge} 2$ \\
\hline 1 & 1.344 .214 & -83.409 & 6.957 .030 .056 & 1.345 .268 & -82.355 & 6.782 .280 .996 \\
\hline 2 & 1.391 .521 & -36.101 & 1.303 .309 .148 & 1.385 .222 & -42.400 & 1.797 .772 .504 \\
\hline 3 & 1.424 .283 & -3.340 & 11.152 .491 & 1.426 .364 & -1.259 & 1.585 .188 \\
\hline 4 & 1.457 .811 & 30.189 & 911.355 .373 & 1.468 .726 & 41.104 & 1.689 .535 .004 \\
\hline 5 & 1.520 .284 & 92.661 & 8.586 .071 .592 & 1.512 .348 & 84.725 & 7.178 .347 .749 \\
\hline 15 & 7.138 .113 & 0 & 17.768 .918 .660 & & & 17.449 .521 .440 \\
\hline
\end{tabular}


52 Retos

Determinación del mercado objetivo y la demanda insatisfecha, cuando no se dispone de estadísticas

\begin{tabular}{|c|c|c|c|c|c|c|}
\hline$\tilde{y}$ & $=$ & $\frac{\sum Y}{n}$ & $=$ & $\frac{7.138 .113}{5}$ & $=$ & 1.427 .623 \\
\hline$R \wedge 2$ & $=$ & $\frac{\sum\left(Y^{\prime}-\tilde{y}\right) \wedge 2}{\sum(Y-\tilde{y}) \wedge 2}$ & $=$ & $\frac{17.449 .521 .440}{17.768 .918 .660}$ & $=$ & 0,9820 \\
\hline$r$ & $=$ & $\sqrt{ } R^{\wedge} 2$ & & & & \\
\hline$r$ & $=$ & 0,90 & & & & \\
\hline
\end{tabular}

PROYECCIÓN DE LA DEMANDA INSATISFECHA

\begin{tabular}{ccc} 
AÑOS & AÑOS $X$ & DEMANDA PROYECTADA Y' \\
\hline 2011 & 6 & 1.557 .264 \\
2012 & 7 & 1.603 .515 \\
2013 & 8 & 1.651 .140 \\
2014 & 9 & 1.700 .178 \\
2015 & 10 & 1.750 .674 \\
\hline
\end{tabular}

Envío 27 de abril de 2011 - aprobación 10 de mayo de 2011 